\title{
Critical Configurations For Radial Distortion Self-Calibration
}

\author{
Changchang $\mathrm{Wu}$ \\ Google Inc.
}

\begin{abstract}
In this paper, we study the configurations of motion and structure that lead to inherent ambiguities in radial distortion estimation (or $3 D$ reconstruction with unknown radial distortions). By analyzing the motion field of radially distorted images, we solve for critical surface pairs that can lead to the same motion field under different radial distortions and possibly different camera motions. We study the properties of the discovered critical configurations and discuss the practically important configurations that often occur in real applications. We demonstrate the impact of the radial distortion ambiguity on multi-view reconstruction with synthetic experiments and real experiments.
\end{abstract}

\section{Introduction}

Structure from motion (SfM) and self-calibration have become commonplace, as recent systems demonstrate automatic 3D reconstructions from large-scale uncalibrated photo collections $[1,3,20]$. These systems show that the self-calibration of unknown radial distortions normally works well. However, it has not been well understood when the self-calibration of radial distortion could fail.

This work was initially motivated by the application of SfM in geographic survey, where Unmanned Aerial Vehicles (UAVs) are used to capture the images. These capture systems typically use wide-angle cameras (e.g. GoPro) in order to cover large areas, and the images may contain significant radial distortions. The captured images are then processed by SfM tools, for example, Bundler [15] and VisualSFM [21, 19], to reconstruct the ground models. While these UAV reconstructions usually work, we find for certain captures that the SfM softwares produce incorrectly distorted 3D models along with incorrect estimations of radial distortions (See Figure 1). These failures in radial distortion estimation inspired this paper.

3D reconstruction is well known to have ambiguities for certain configurations of motion and structure. These so called critical configurations have been extensively studied, for example, for Euclidean reconstruction with calibrated cameras $[5,12,9,7]$ and self-calibration of linear cameras $[17,18,6,10]$. In real applications, cameras often have significant radial distortions that need to be explicitly modeled. However, we find little previous study on the critical configurations for radial distortion. In this paper, we will study the configurations that can lead to ambiguous reconstructions due to the ambiguity of radial distortion. Our main contributions are the following:

- A motion field based framework for solving ambiguous configurations under a general radial distortion model;

- We solve for the critical surfaces and motions for radial distortion self-calibration using the new framework;

- We present an important critical configuration that often occurs in practical applications.

Our findings can guide image capture to better avoid ambiguous radial distortion estimation, and correspondingly show when pre-calibration of radial distortion is necessary.

The remainder is organized as follows: Section 2 discusses the background of the critical configuration study. Section 3 introduces our framework for analyzing the ambiguities for radial distortion self-calibration. We investigate the critical configurations and their properties in Section 4 and 5, and then discuss in Section 6 a practically important configuration that exists under known motion directions. Conclusions and future work are given in Section 7.

\section{Background}

In the past several decades, researchers have investigated the critical configurations for many different 3D reconstruction problems. Given any camera motion, there may exist a family of possible critical surfaces for which $3 \mathrm{D}$ reconstruction is ambiguous (regardless of the scale). Ruled quadrics or their degenerate forms are critical surfaces for two view reconstruction and image velocity based reconstruction [11, 13, 5, 12]. For three views or more, critical elliptic quartics may exist for Euclidean reconstruction and projective reconstruction [9, 4, 8, 7]. For the self-calibration of linear cameras, there exist a set of critical motions under which 3D reconstruction is ambiguous regardless of the structures. A complete study of such critical 

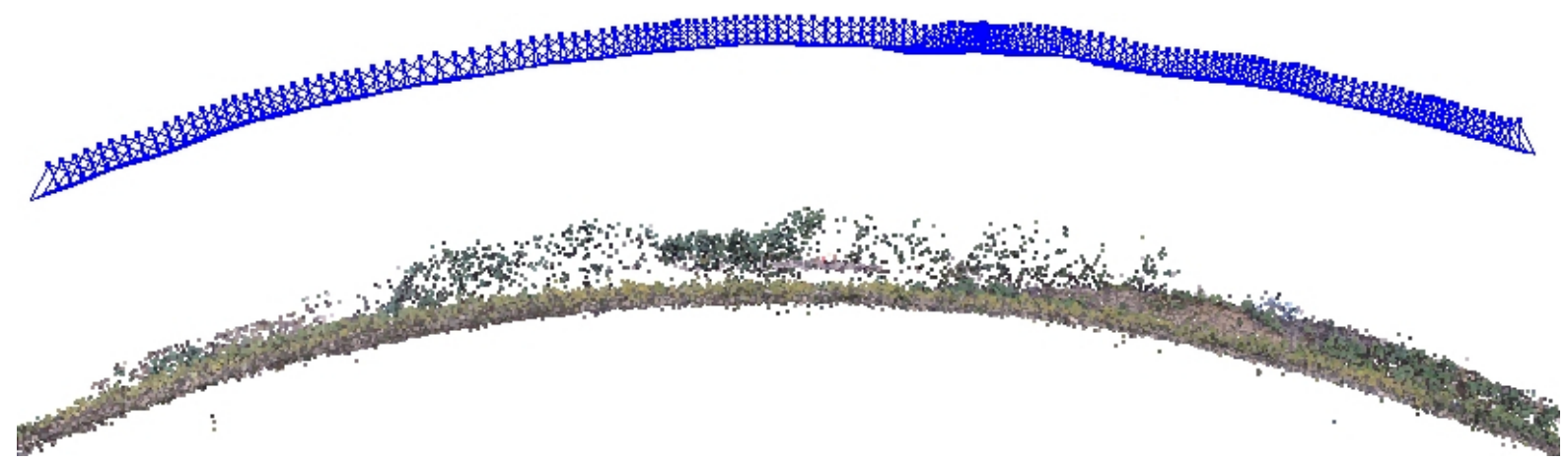

(a) A critical configuration that leads to a significantly distorted reconstruction (of the roughly flat ground). As verified by the undistorted images, the radial distortion is not correctly estimated. See Figure 3 where we re-produce the same problem using a synthetic dataset.

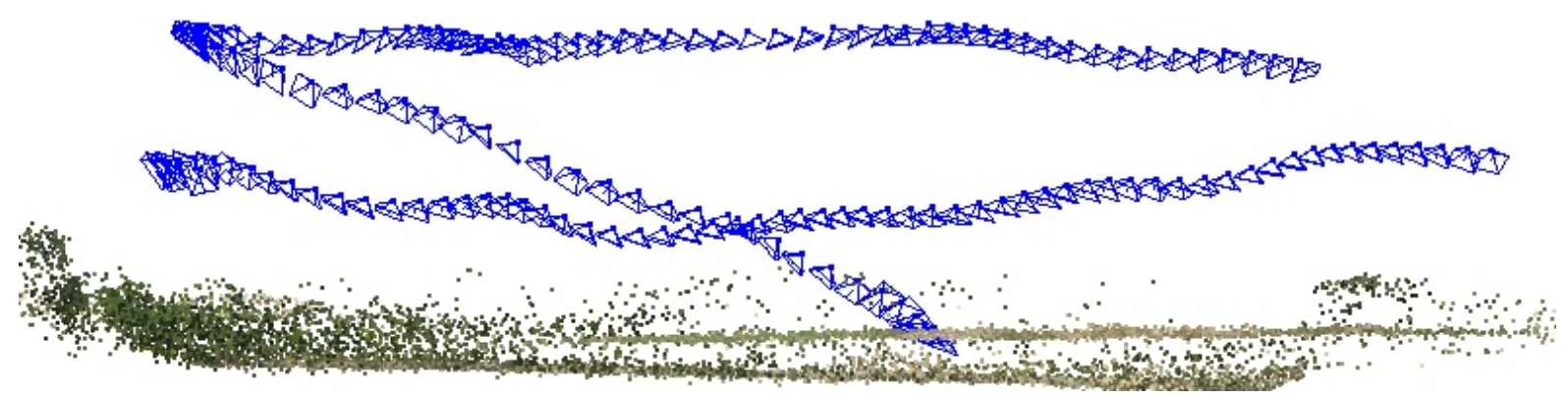

(b) A typical successful reconstruction example, where radial distortions are well estimated. Notice one of the flat ground surface.

Figure 1. Reconstruction of two GoPro UAV sequences using VisualSFM [19] (similar results are produced by Bundler [15]). No radial distortion calibration is specified to the software. As shown by the blue pyramids, the camera points straight downward and moves parallel to the ground in the first capture. In the second capture, the camera has more variations in moving directions and viewing directions.

motions for monocular self-calibration has been presented by Sturm [17], and further study for varying intrinsics can be found in $[18,10]$. However, no prior work has examined the critical configurations for radial distortion. In this paper, we will study this new critical configuration problem.

A few special degenerate configurations for radial distortion self-calibration have been reported previously. For example, Mičušík et al. [14] and Brito et al. [2] recognize forward motion as a degenerate case for recovering radial distortion parameters. Additionally, Mičušík et al. [14] find pure translation to be ambiguous if all point displacements are parallel to the camera's translation in XY plane. These are in fact special cases. This paper will present a full analysis of the critical configurations for arbitrary motions.

Although modern reconstruction algorithms use discrete point correspondences instead of motion fields, we find the latter more convenient for analyzing radial distortions. Unlike the complicated non-linear relationship between the radial distortions in discrete camera frames, the radial distortion in a continuous motion can be conveniently modeled with derivatives, which allows to solve for the critical configurations without any explicit parametrization. In this paper, we opt for the differential approach, in particular, on top of the motion field study by Horn [5]. As deferential motions correspond to small discrete camera motions, our findings are valuable for the reconstruction of video sequences, where the relative ambiguities can accumulate to large reconstruction errors.

\section{Problem formulation}

The question we want to answer is the following: given two cameras with different radial distortions and possibly different motions, what surfaces could lead to the same motion field? Such surfaces would be critical for radial distortion self-calibration. Accordingly, the family of ambiguous motion fields is given by the possible configurations of camera pairs and their corresponding critical surfaces.

To focus on the ambiguity caused by radial distortions alone, we assume the two cameras to have the same linear intrinsics. As reviewed earlier, the ambiguity of the linear intrinsics has already been well studied. If the analysis includes more varying parameters, such as focal length, the ambiguity would be a larger super-set of the radial distortion related ambiguity, the study of which is beyond the scope of this paper.

\subsection{Linear projection}

First, we briefly review the motion field of linear cameras using notations similar to [5]. Without loss of generality, we 
consider the image of an identity camera. The projection $p=(x, y, 1)^{T}$ of a $3 \mathrm{D}$ point $P=(X, Y, Z)^{T}$ is

$$
p=\frac{1}{P \cdot \hat{z}} P,
$$

where $\hat{z}=(0,0,1)^{T}$ is the viewing direction. Given a relative moving speed $P^{\prime}$, the velocity of its observation is

$$
p^{\prime}=\frac{1}{P \cdot \hat{z}}\left(P^{\prime}-\left(P^{\prime} \cdot \hat{z}\right) p\right) .
$$

Suppose the camera moves with an instantaneous translational velocity $t$ and an instantaneous rotational velocity $\omega$, the velocity of the $3 \mathrm{D}$ point relative to the camera is

$$
P^{\prime}=-t+P \times \omega=-t+(P \cdot \hat{z}) p \times \omega .
$$

By substituting $P^{\prime}$, we obtain the image velocity

$$
p^{\prime}=\frac{1}{Z}((t \cdot \hat{z}) p-t)+[p, \omega, \hat{z}] p-p \times \omega,
$$

where $[p, \omega, \hat{z}]=p \cdot(\omega \times \hat{z})$ denotes the triple product.

\subsection{General radial distortion}

Now we consider the images with radial distortion. With a typical assumption of the radial distortion being central and centered around the principal point, each image circle around the principal point corresponds to another rescaled image circle in the undistorted image. With this simple mechanism in mind, we use a parameter-free radial distortion model based on the scaling of the image circles. Let $f\left(r^{2}\right)$ be the scaling factor for the image circle $r^{2}=$ $x^{2}+y^{2}=\left(p^{T} p-1\right)$, the coordinate mapping from the distorted image to the undistorted image can be written as:

$$
\operatorname{undistort}(p)=\operatorname{diag}\left(f\left(r^{2}\right), f\left(r^{2}\right), 1\right) p .
$$

We will refer to $f\left(r^{2}\right)$ as the radial distortion function. For convenience, we define two helper matrix functions

$$
F\left(r^{2}\right)=\operatorname{diag}(f, f, 1) \text { and } F^{\prime}\left(r^{2}\right)=\operatorname{diag}\left(f^{\prime}, f^{\prime}, 0\right) .
$$

In general, we have $f^{\prime}\left(r^{2}\right) \neq 0$ because $f\left(r^{2}\right)$ should not be a constant. From now on, we will omit the argument $\left(r^{2}\right)$ for $f, f^{\prime}, F$, and $F^{\prime}$ to simplify the equations, so the undistorted coordinate can be simply written as $F p$.

This parameter-free radial distortion model avoids the possible limitations and complexities of explicit parametrization. As a result, our findings will not be limited to any specific radial distortion parametrization, and can be applied to both typical near-linear cameras and central omni-directional cameras.

It is worth pointing out that $f(0)$ would correspond to the reciprocal of focal length if we also model the variation of focal length. Although real radial distortions have $f(0)=1$, this paper will solve the critical configurations without explicit assumptions on $f(0)$. While our analysis focuses on just radial distortion, the discovered configurations may be applied to additional variations of focal length.

\subsection{Projection under radial distortion}

Given a point $p$ in a distorted image, we can obtain the velocity of the undistorted image point $F p$ by substituting $p$ with $F p$ into Equation 1:

$(F p)^{\prime}=\frac{1}{Z}((t \cdot \hat{z}) F p-t)+[F p, \omega, \hat{z}](F p)-(F p) \times \omega$.

In addition, we know the relationship between the velocity $p^{\prime}$ in the distorted image and the velocity $(F p)^{\prime}$ in the undistorted image from differentiation:

$$
(F p)^{\prime}=F p^{\prime}+F^{\prime}\left(p^{T} p\right)^{\prime} p=\left(F+2 F^{\prime} p p^{T}\right) p^{\prime} .
$$

The above two forms of $(F p)^{\prime}$ must be equal to each other.

\subsection{Ambiguous radial distortion}

Suppose a distortion-free camera with motion $\left\{t_{1}, \omega_{1}\right\}$ along with depth $Z_{1}(x, y)$ produces the same motion field as does a motion $\left\{t_{2}, \omega_{2}\right\}$ along with depth $Z_{2}(x, y)$ under a radial distortion function $f$, the following holds true:

$$
\begin{aligned}
& \frac{\left(t_{2} \cdot \hat{z}\right) F p-t_{2}}{Z_{2}}+\left[F p, \omega_{2}, \hat{z}\right](F p)-(F p) \times \omega_{2}= \\
& \left(F+2 F^{\prime} p p^{T}\right)\left(\frac{\left(t_{1} \cdot \hat{z}\right) p-t_{1}}{Z_{1}}+\left[p, \omega_{1}, \hat{z}\right] p-p \times \omega_{1}\right) .
\end{aligned}
$$

Since the Z-component of the image velocity is always 0 , the vector equality gives two scalar constraints, which are usually sufficient for solving $Z_{1}(x, y)$ and $Z_{2}(x, y)$. Such a configuration of $\left\{t_{1}, t_{2}, \omega_{1}, \omega_{2}, f, Z_{1}, Z_{2}\right\}$ is called a critical configuration, where $Z_{1} p$ and $Z_{2} F p$ are the critical surface pair for $\left\{t_{1}, t_{2}, \omega_{1}, \omega_{2}, f\right\}$. In the next Section, we will use this constraint to solve for the critical surfaces and derive critical motions.

The configuration with one distortion-free camera does not lose any generality in terms of critical surfaces. We can apply the undistortion transformation of the second camera to produce the undistorted image, for which the first camera can be seen as the distorted one. Similarly, given two cameras that have different radial distortion functions, we can apply one of the undistortion transformations and produce the configuration of one distortion-free camera and another camera with a relative distortion.

\section{Critical configurations}

In this section, we solve for the critical surfaces and then derive the critical motions where any surface is critical.

\subsection{Critical surfaces}

For a moment we will assume $t_{2} \neq 0$. By taking the dot-product of the Equation 2 with $t_{2} \times F p$, we can 
eliminate $Z_{2}$ and obtain the constraint for solving the first critical surface $Z_{1}$ as follows

$$
\begin{aligned}
& 0=\left((F p) \times \omega_{2}-F\left(p \times \omega_{1}\right)\right) \cdot\left(t_{2} \times F p\right) \\
& +\frac{2}{Z_{1}}\left(\left(t_{1} \cdot \hat{z}\right)\left(p^{T} p\right)-p^{T} t_{1}\right)\left(F^{\prime} p\right) \cdot\left(t_{2} \times F p\right) \\
& +\frac{1}{Z_{1}}\left[t_{2}, F t_{1}, F p\right]+2\left(p^{T} p\right)\left[p, \omega_{1}, \hat{z}\right]\left(F^{\prime} p\right) \cdot\left(t_{2} \times F p\right) .
\end{aligned}
$$

Given $F p=\left(f / f^{\prime}\right)\left(F^{\prime} p\right)+\hat{z}$, we find $\left(F^{\prime} p\right) \cdot\left(t_{2} \times F p\right)=$ $\left(F^{\prime} p\right) \cdot\left(t_{2} \times \hat{z}\right)=f^{\prime}\left(t_{2} \times \hat{z}\right)^{T} p$. By further expanding the triple products, we can rewrite the above equation as

$$
\begin{aligned}
& 0=\left((F p) \times \omega_{2}-F\left(p \times \omega_{1}\right)\right) \cdot\left(t_{2} \times F p\right) \\
& +\frac{2 f^{\prime}}{Z_{1}}\left(t_{1} \cdot \hat{z}\right)\left(p^{T} p\right)\left(t_{2} \times \hat{z}\right)^{T} p-\frac{2 f^{\prime}}{Z_{1}} p^{T} t_{1}\left(t_{2} \times \hat{z}\right)^{T} p \\
& +\frac{1}{Z_{1}}\left(t_{2} \times F t_{1}\right)^{T} F p+2 f^{\prime}\left(p^{T} p\right) p^{T}\left(\omega_{1} \times \hat{z}\right)\left(t_{2} \times \hat{z}\right)^{T} p
\end{aligned}
$$

which defines the first critical surface $Z_{1} p$. Another way to view this critical surface is a depthmap:

$$
Z_{1}=\frac{\left(\begin{array}{c}
-2 f^{\prime}\left(t_{1} \cdot \hat{z}\right)\left(p^{T} p\right)\left(t_{2} \times \hat{z}\right)^{T} p+ \\
2 f^{\prime} p^{T} t_{1}\left(t_{2} \times \hat{z}\right)^{T} p-\left(t_{2} \times F t_{1}\right)^{T} F p
\end{array}\right)}{\left(\begin{array}{c}
\left((F p) \times \omega_{2}-F\left(p \times \omega_{1}\right)\right) \cdot\left(t_{2} \times F p\right) \\
+2 f^{\prime}\left(p^{T} p\right) p^{T}\left(\omega_{1} \times \hat{z}\right)\left(t_{2} \times \hat{z}\right)^{T} p
\end{array}\right)}
$$

The depth from such a division is obviously valid only when the denominator is non-zero. If both the numerator and the denominator are zero, any depth satisfies Equation 3 , so the viewing ray would lie on the critical surface. In case only the denominator is zero, the resulting depth would be infinite, where the image point is a vanishing point of the critical surface.

By taking the dot-product of Equation 2 with $\hat{z} \times p$, we find a simple relationship between the critical surface pair:

$$
\begin{aligned}
\frac{1}{Z_{2}} t_{2} \cdot(\hat{z} \times p) & -\frac{1}{Z_{1}}\left(F t_{1}\right) \cdot(\hat{z} \times p)= \\
& -\left((F p) \times \omega_{2}-F\left(p \times \omega_{1}\right)\right) \cdot(\hat{z} \times p)
\end{aligned}
$$

which allows us to obtain $Z_{2}$ from $Z_{1}$ when $t_{2} \times \hat{z} \neq 0$. The depthmap of the second critical surface can be obtained as:

$$
Z_{2}=\frac{Z_{1} t_{2} \cdot(\hat{z} \times p)}{\left(F t_{1}-\left((F p) \times \omega_{2}-F\left(p \times \omega_{1}\right)\right) Z_{1}\right) \cdot(\hat{z} \times p)} .
$$

The second critical surface is then given by $Z_{2} F p$. Note when $t_{2} \times \hat{z}=0$, we can still solve for $Z_{2}$ by taking dot-product with $p$ instead, which this paper will skip.

Figure 2 demonstrates some interesting critical surfaces and their motion fields. The critical surfaces yield the motion fields that are ambiguous for radial distortion selfcalibration. Despite the difference of using image velocity here, the degenerate case found by [14] is apparently only a special case. We will discuss more properties of the critical configurations in Section 5.

\subsection{Critical motions}

There exist critical motions under which any surface is ambiguous for recovering radial distortions. For any critical motion, Equation 2 and 3 must hold true for any depth $Z_{1}$ and $Z_{2}$, any image point $p$, and any radial distortion, therefore the following two constraints must be satisfied:

$$
\begin{aligned}
0= & \left((F p) \times \omega_{2}-F\left(p \times \omega_{1}\right)\right) \cdot\left(t_{2} \times F p\right) \\
& +2 f^{\prime}\left(p^{T} p\right) p^{T}\left(\omega_{1} \times \hat{z}\right)\left(t_{2} \times \hat{z}\right)^{T} p \\
0= & 2 f^{\prime}\left(t_{1} \cdot \hat{z}\right)\left(p^{T} p\right)\left(t_{2} \times \hat{z}\right)^{T} p \\
& -2 f^{\prime} p^{T} t_{1}\left(t_{2} \times \hat{z}\right)^{T} p+\left(t_{2} \times F t_{1}\right)^{T} F p .
\end{aligned}
$$

The two equations can be viewed as polynomial functions of $p, f$, and $f^{\prime}$, where the coefficient for each term of different order must be zero.

Consider firstly the general case of $t_{1} \neq 0$ and $t_{2} \neq 0$, Equation 8 requires $t_{1} \times \hat{z}=t_{2} \times \hat{z}=0$, and Equation 7 then requires $\omega_{1} \times \hat{z}=\omega_{2} \times \hat{z}=0$ and $\omega_{1}=\omega_{2}$. That is, translation along the optical axis and rotation around the optical axis are critical motions for recovering radial distortion. Note the critical motion includes the combination of them.

When $t_{1}=0$ or $t_{2}=0$, we find no real critical surfaces, except for the critical motion of rotation around the optical axis. If $t_{1}=0$ and $t_{2} \neq 0$, we are still able to obtain Equation 7 from Equation 3, which leads to $\omega_{1} \times \hat{z}=\omega_{2} \times \hat{z}=0$ and $\omega_{1}=\omega_{2}$. By applying these conditions in Equation 2, we find $\left(t_{2} \cdot \hat{z}\right) F p-t_{2}=0$, which can be satisfied for all $p$ only when $t_{2}=0$. Similarly we must have $t_{1}=0$ when $t_{2}=0$. Therefore, radial distortion is not ambiguous for pure rotations unless the rotation is around the optical axis.

In summary, for the self-calibration of radial distortion, we find the following critical motions:

$$
t \times \hat{z}=\omega \times \hat{z}=0 .
$$

The ambiguity is not surprising since such motions are symmetric around the optical axis. The forward motion degeneracy reported in $[14,2]$ is a special case when $\omega=0$. On the other hand, these critical motions is a subset of the critical motions for the linear camera self-calibration [17]. In further analysis of critical surfaces, we will exclude the critical motions and any pure rotation cameras.

\section{Properties of the critical configurations}

This section discusses a few properties of the critical configurations for radial distortion self-calibration.

\subsection{Comparison with the distortion-free case}

It can be seen from Equation 3 and 4 that the critical surfaces for radial distortion self-calibration have high degrees depending on the radial distortion function $f$. In comparison, the critical surfaces for the distortion-free 


\section{$Z_{1}$}

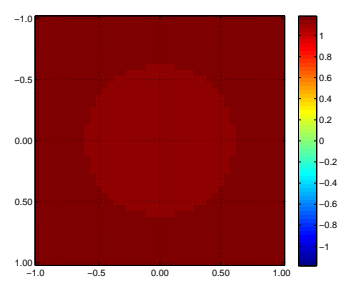

$Z_{2}$
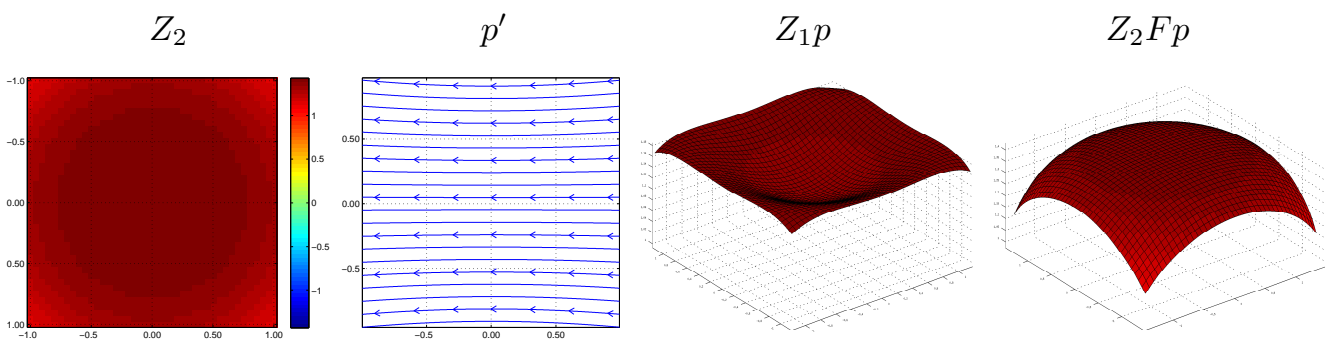

(a) $t_{1}=t_{2}=(1,0,0)^{T}, \omega_{1}=(0,-0.1,0)^{T}, \omega_{2}=(0,-0.3,0)^{T}$. The critical surfaces are rotationally symmetric. Note the convexity/concavity.
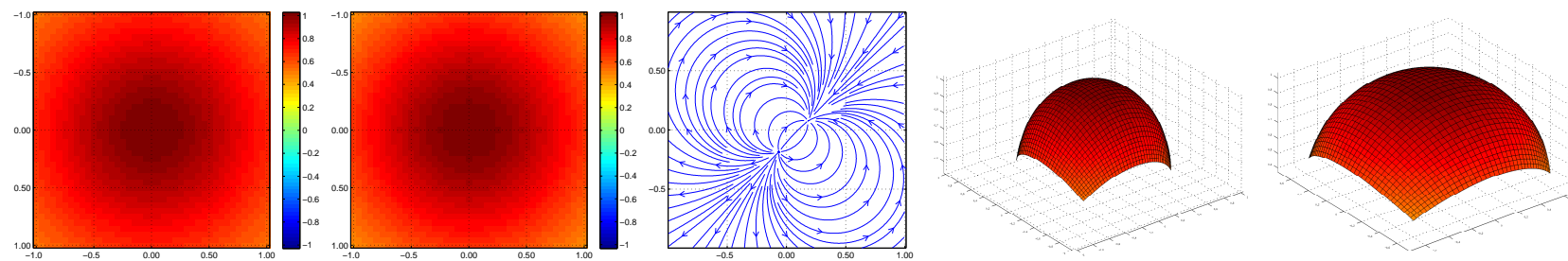

(b) $t_{1}=t_{2}=(-0.683853,-0.72962,0.0)^{T}, \omega_{1}=(0.695244,-0.6516340,-0.0766849)^{T}, \omega_{2}=(0.692557,-0.649115,-0.0766849)^{T}$. The critical surfaces are rotationally symmetric. The motion field is similar to a magnetic field instead of being roughly parallel.


(c) $t_{1}=t_{2}=(-0.01,0,-1)^{T}, \omega_{1}=(0,0.01,0.05)^{T}, \omega_{2}=(0,0.099,0.05)^{T}$. The translations are close to being forward in this example.
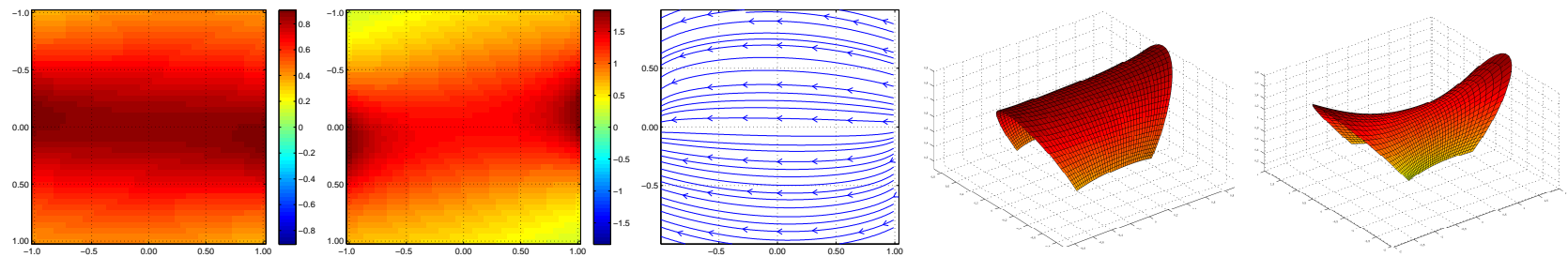

(d) $t 1=(0.850138,-0.526560,0)^{T}, t_{2}=(0.992789,-0.119871,0)^{T}, \omega_{1}=(0.600179,0.419835,0)^{T}, \omega_{2}=(0.080059,0.179102,0)^{T}$.
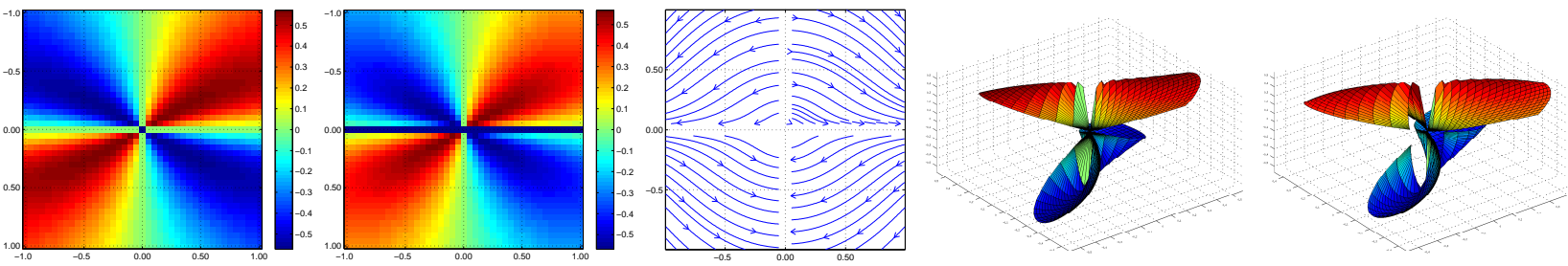

(e) $t_{1}=t_{2}=\omega_{1}=\omega_{2}=(1,0,0)^{T}$. Instead of being perpendicular, the translation and rotation are parallel in this example.
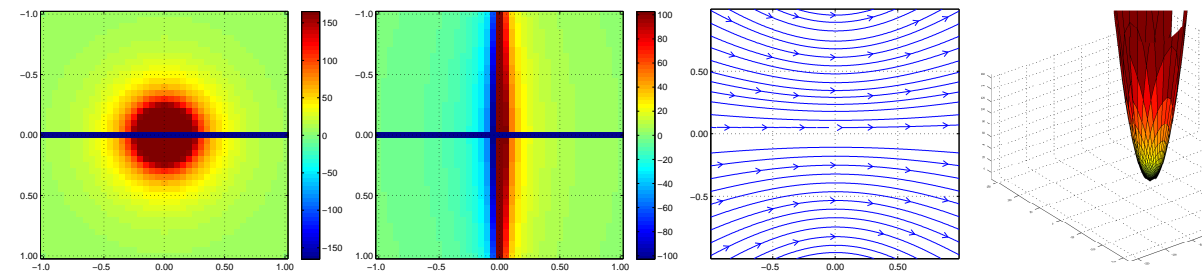

(f) $t_{1}=(1,0,0)^{T}, t 2=(0,0,1)^{T}, \omega_{1}=\omega_{2}=(0,1,0)^{T}$. The second camera has forward motion, and the first surface is rotationally symmetric.

Figure 2. Critical surface examples created with identity cameras and a radial distortion function: $f\left(r^{2}\right)=1+0.1 r^{2}+0.012 r^{4}$. From left to right are 1) the first depthmap, 2) the second depthmap, 3) motion field direction, 4) first critical surface, 5) second critical surface. Note only the sub-portion of the critical surface corresponding to the $[-1,1] \times[-1,1]$ image region is visualized. 
problem can be obtained by setting $f=1$ and $f^{\prime}=0$, which become the well-known simpler ruled quadrics:

$$
\left(P \times\left(\omega_{2}-\omega_{1}\right)\right) \cdot\left(t_{2} \times P\right)+\left(t_{2} \times t_{1}\right)^{T} P=0 .
$$

For many camera configurations, the critical surface for radial distortion self-calibration is in fact similar to the corresponding ruled quadrics, and can be seen as a distorted version of the counterpart. An example configuration with such critical surfaces can be found in Figure 2(d).

When the camera motion is known to be pure translation $\omega_{1}=\omega_{2}=0$ or pure rotation $t_{1}=t_{2}=0$, critical surfaces do not exist in the distortion-free case. When radial distortion is considered, there exist the previously discussed critical motions but no additional critical surfaces. It is already shown that radial distortion is not ambiguous for pure rotation except for rotations around the optical axis. Similarly, radial distortion is found not ambiguous for pure translation except when it is along the optical axis.

The biggest difference regards the case of known rotation $\omega_{1}=\omega_{2} \neq 0$ and known translation $t_{1}=t_{2} \neq 0$ (ignoring scale). Under such conditions, it is known that critical surfaces do not exist for the distortion-free problem [5]. In contrast for radial distortion self-calibration, critical surfaces still exist, even when both rotation and translation are known: $t_{1}-t_{2}=\omega_{1}-\omega_{2}=0$. This can be seen from Equation 4 because $f^{\prime} \neq 0$. See Figure 2 for examples of such critical surfaces.

The new kind of critical surfaces are particularly interesting. It shows that $3 \mathrm{D}$ reconstruction with unknown radial distortion may still be ambiguous even when translation and (or) rotation are correctly estimated.

\subsection{Additional rotation around the optical axis}

For any configuration of motion and surface that satisfies Equation 2, let us consider an additional rotation velocity around the optical axis such that $\omega_{1}^{\delta}=\omega_{1}+\delta \hat{z}$ and $\omega_{2}^{\delta}=\omega_{2}+\delta \hat{z}$. The addition to the left side of Equation 2 is $(F p) \times(\delta \hat{z})=f \delta(p \times \hat{z})$, and is equal to the addition to the right side $F(p \times(\delta \hat{z}))=f \delta(p \times \hat{z})$. Therefore, Equation 2 still holds for $\left\{t_{1}, \omega_{1}^{\delta}, t_{2}, \omega_{2}^{\delta}\right\}$ with the same $Z_{1}$ and $Z_{2}$. That is, equal additions to the rotation velocities around the optical axis does not change the critical surfaces, despite the changes in the motion fields.

\section{An interesting degenerate case}

As shown by the polynomial terms of different degrees in Equation 3, critical surfaces are complicated in general. We are interested in possible common divisors for those terms, so that the depth in Equation 4 can be simplified.

\subsection{A common polynomial divisor}

An interesting configuration arises during our analysis. We find Equation 3 of the first critical surface has a second order polynomial factor as follows:

$$
\Phi=p^{T} t_{1}\left(t_{2} \times \hat{z}\right)^{T} p,
$$

when the following conditions are met:

$$
\begin{aligned}
& t_{1} \cdot \hat{z}=t_{2} \cdot \hat{z}=0, t_{1} \times t_{2}=0, \text { and } t_{1} \cdot t_{2} \neq 0 \\
& \left(\omega_{1}-\omega_{2}\right) \cdot \hat{z}=0 \text { and } t_{1} \cdot \omega_{1}=t_{2} \cdot \omega_{2}=0 .
\end{aligned}
$$

The camera motions can be viewed as the differential mode of one of the following well known cases:

- Moving on a sphere while pointing to center [16]. Orbital motion with additional rotation around the optical axis.

- Moving on a plane that is perpendicular to the viewing direction. This can be seen as moving on an infinite sphere $(\omega \times \hat{z}=0)$ in the above case.

It can be seen that $t_{1}, t_{2},\left(\omega_{1} \times \hat{z}\right)$, and $\left(\omega_{2} \times \hat{z}\right)$ are parallel. Note critical surfaces will not exist for the distortion-free problem due to the known translation $t_{1} \times t_{2}=0$.

We first try to simplify the cross products that involve $F p$, that is, $(F p) \times \omega_{2}-F\left(p \times \omega_{1}\right)$. Using the invariance discussed in Section 5.2, we can assume $\omega_{1} \cdot \hat{z}=\omega_{2} \cdot \hat{z}=0$ from $\left(\omega_{1}-\omega_{2}\right) \cdot \hat{z}=0$ without changing the critical surfaces. By using the fact $p=(p-\hat{z})+\hat{z}$, we find

$$
\begin{aligned}
& (F p) \times \omega_{2}-F\left(p \times \omega_{1}\right) \\
& =f(p-\hat{z}) \times \omega_{2}+\hat{z} \times \omega_{2}-(p-\hat{z}) \times \omega_{1}-f\left(\hat{z} \times \omega_{1}\right) \\
& =(p-\hat{z}) \times\left(f \omega_{2}-\omega_{1}\right)+\hat{z} \times\left(\omega_{2}-f \omega_{1}\right),
\end{aligned}
$$

Under the proposed conditions, we can prove the terms in the numerator and the denominator of Equation 4 divisible by $\Phi$. By removing the dot-products of the perpendicular terms that produce zeros and by expanding the quadruple product, we can transform as follows:

$$
\begin{aligned}
& \left((F p) \times \omega_{2}-F\left(p \times \omega_{1}\right)\right) \cdot\left(t_{2} \times F p\right) \\
& =\left(\begin{array}{c}
(p-\hat{z}) \times\left(f \omega_{2}-\omega_{1}\right) \\
+\hat{z} \times\left(\omega_{2}-f \omega_{1}\right)
\end{array}\right) \cdot\left(\begin{array}{c}
f t_{2} \times(p-\hat{z}) \\
+t_{2} \times \hat{z}
\end{array}\right) \\
& =\left((p-\hat{z}) \times\left(f \omega_{2}-\omega_{1}\right)\right) \cdot\left(f t_{2} \times(p-\hat{z})\right) \\
& =\left((p-\hat{z}) \cdot t_{2}\right)\left((p-\hat{z}) \cdot\left(f^{2} \omega_{2}-f \omega_{1}\right)\right) \\
& =-p^{T}\left(\left(f^{2} \omega_{2}-f \omega_{1}\right) \times \hat{z}\right)\left(t_{2} \times \hat{z}\right)^{T} p,
\end{aligned}
$$

which can be divided by $\Phi$ because $t_{1}\left\|\left(\omega_{1} \times \hat{z}\right)\right\|\left(\omega_{2} \times \hat{z}\right)$. Similarly, the term $2 f^{\prime}\left(p^{T} p\right) p^{T}\left(\omega_{1} \times \hat{z}\right)\left(t_{2} \times \hat{z}\right)^{T} p$ in the denominator can be divided by $\Phi$ because $t_{1} \|\left(\omega_{1} \times \hat{z}\right)$. As for the terms in the numerator, both $\left(t_{1} \cdot \hat{z}\right)\left(p^{T} p\right)\left(t_{2} \times \hat{z}\right)^{T} p$ and $\left(t_{2} \times F t_{1}\right)^{T} F p$ become zero, and the only left term is $2 f^{\prime} p^{T} t_{1}\left(t_{2} \times \hat{z}\right)^{T} p=2 f^{\prime} \Phi$.

The common divisor $\Phi$ itself defines two critical planes $t_{1}^{T} p=0$ and $\left(t_{2} \times \hat{z}\right)^{T} p=0$. The two planes are not too interesting because the camera center is on the planes and they are also perpendicular to the image plane. We will leave the planes here and focus on the simplified depth. 


\subsection{Rotationally symmetric surfaces}

By using the common divisor $\Phi$, the first critical surface becomes much simplified

$$
\begin{aligned}
Z_{1}= & \frac{2 f^{\prime} p^{T} t_{1}\left(t_{2} \times \hat{z}\right)^{T} p}{\left(\begin{array}{c}
-p^{T}\left(\left(f^{2} \omega_{2}-f \omega_{1}\right) \times \hat{z}\right)\left(t_{2} \times \hat{z}\right)^{T} p \\
+2 f^{\prime}\left(p^{T} p\right) p^{T}\left(\omega_{1} \times \hat{z}\right)\left(t_{2} \times \hat{z}\right)^{T} p
\end{array}\right)} \\
= & \frac{2\left(t_{1} \cdot t_{2}\right) f^{\prime}}{\left(\begin{array}{c}
-\left(t_{2} \cdot\left(\omega_{2} \times \hat{z}\right)\right) f^{2}+\left(t_{2} \cdot\left(\omega_{1} \times \hat{z}\right)\right) f \\
+2\left(t_{2} \cdot\left(\omega_{1} \times \hat{z}\right)\right)\left(p^{T} p\right) f^{\prime}
\end{array}\right)},
\end{aligned}
$$

which gives a single depth value for each image circle $r^{2}=x^{2}+y^{2}=p^{T} p-1$. The resulting critical surface is rotationally symmetric around the optical axis.

The second critical surface can be solved correspondingly using the relationship between the two depthmaps. Equation 13 allows the the following simplification:

$$
\begin{array}{r}
\left((F p) \times \omega_{2}-F\left(p \times \omega_{1}\right)\right) \cdot(\hat{z} \times p) \\
=\left(\hat{z} \times\left(\omega_{2}-f \omega_{1}\right)\right) \cdot(\hat{z} \times p) .
\end{array}
$$

Accordingly, the second critical surface is simplified to

$$
\begin{aligned}
Z_{2} & =\frac{Z_{1} t_{2} \cdot(\hat{z} \times p)}{\left(F t_{1}-Z_{1}\left(\hat{z} \times\left(\omega_{2}-f \omega_{1}\right)\right)\right) \cdot(\hat{z} \times p)} \\
& =\frac{\left(t_{1} \cdot t_{2}\right) Z_{1}}{\left(t_{1} \cdot t_{1}\right) f-t_{1} \cdot\left(\hat{z} \times\left(\omega_{2}-f \omega_{1}\right)\right) Z_{1}},
\end{aligned}
$$

which is again rotationally symmetric. ${ }^{1}$ Two examples of such critical surface are given in Figure 2(a) and 2(b). The pair of critical surfaces are both rotationally symmetric, but have different curvatures and even different curvature signs.

For a limited camera viewing angle, the portion of visible critical surfaces can be near-spherical, near-planar, or even a perfect plane depending on the motion and radial distortion function. For example, when $f\left(r^{2}\right)=1 /\left(1-\lambda r^{2}\right)$ and $\omega_{1} \times \hat{z}=0$, we are given a constant depth $Z_{1}=\frac{4 \lambda\left(t_{1} \cdot t_{2}\right)}{t_{2} \cdot\left(\hat{z} \times \omega_{2}\right)}$. Let us revisit the UAV capture in Figure 1(a), where the camera moves parallel to the ground and the camera points to the ground. The visible surface relative to each camera is near-planar and thus have caused the ambiguity in radial distortion estimation.

\subsection{Experiments}

We devise two synthetic image sequences to verify the radial distortion ambiguity and demonstrate its impact on multi-view 3D reconstruction. We feed noise-free feature

\footnotetext{
${ }^{1}$ There exist other configurations where only one critical surface is rotationally symmetric. For example, when $t_{1} \cdot \hat{z}=\left(\omega_{1}-\omega_{2}\right) \cdot \hat{z}=$ $t_{1} \cdot \omega_{1}=t_{1} \cdot \omega_{2}=0$ and the second camera moves forward $t_{2} \times \hat{z}=0$, only the first critical surface is rotationally symmetric $Z_{1}=\frac{t_{1} \cdot t_{1}}{t_{1} \cdot\left(\hat{z} \times\left(\omega_{2}-f \omega_{1}\right)\right)}$. See Figure 2(f) for an example.
}

coordinates and perfect matches to VisualSFM [19], and compare the automatically reconstructed models to the ground-truth models. Although the reconstruction method is not velocity-based, we expect the same ambiguity with densely sampled image sequences.

First, as shown in Figure 3, we move a camera above a planar point grid at a constant height and keep the camera pointing to the plane. The distorted images are generated for the radial distortion $f\left(r^{2}\right)=1+\lambda r^{2}$, where $\lambda=0.2$. This can be seen as an ideal version of the capture in Figure 1(a). Similar to the real capture, the automatic reconstruction of the synthetic dataset produces an incorrect distorted 3D model due to the ambiguity of radial distortion.

Second, we use a point grid on a perfect sphere. The camera moves on a co-centered outer sphere and points to the sphere center. The distorted images are generated for the radial distortion $f\left(r^{2}\right)=1+\lambda r^{2}$, where $\lambda=-0.2$. The spherical surface is rotationally symmetric and ambiguous for the reconstruction. As shown in Figure 4, the automatic reconstruction produces a concave surface instead.

The experiments show that multi-view reconstruction can fail to self-calibrate radial distortions under certain critical configurations. The distortion of the reconstruction is caused locally by the curvature difference between the critical surface pairs and globally by the curvature error accumulation from the persistent ambiguity in the entire capture.

Typical SfM systems initialize the radial distortions of new cameras to zero and rely on bundle adjustments to optimize the parameters. We observe a tendency to under-estimate the radial distortions in near-degenerate configurations, which can be partially explained by the zero initialization. The ambiguity of radial distortion is likely to cause significant inaccuracy when the radial distortions are sever or when the initial estimations are too inaccurate. Also note there would be more ambiguity when using more radial distortion parameters, for example, $f\left(r^{2}\right)=1+\lambda_{1} r^{2}+\lambda_{2} r^{4}$ compared to $f\left(r^{2}\right)=1+\lambda_{1} r^{2}$.

\section{Conclusions and future work}

This paper presents the critical configurations for radial distortion self-calibration under a general radial distortion model. It is shown that radial distortion introduces a new kind of ambiguity into SfM. Unlike the pure linear camera parametrization, critical surfaces exist even for known translations and known rotations due to radial distortion. In particular, this paper demonstrates the practically important critical configurations that should be avoided in real capture for radial distortion self-calibration.

This paper is not meant to be a complete study of all possible ambiguities related to radial distortion. The author wishes to conduct a numerical stability study on neardegenerate congurations and extend the investigation from continuous motion to discrete viewpoints in the future. 

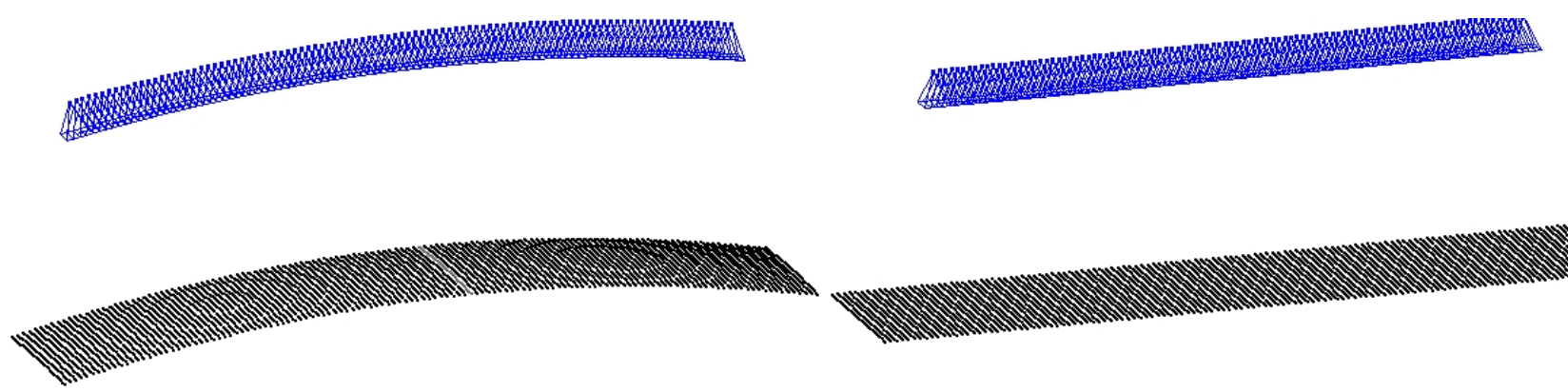

(a) The automatic reconstruction estimates an incorrect $\lambda^{\prime}=0.02$

(b) The ground-truth reconstruction using the known $\lambda=0.2$

Figure 3. Reconstruction of a planar point grid. The blue pyramids are the cameras and the black dots are the reconstructed points.



(a) The automatic reconstruction estimates an incorrect $\lambda^{\prime}=0.025$

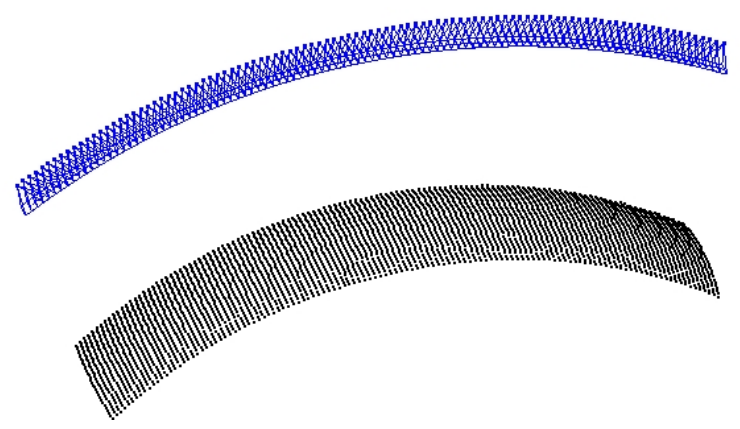

(b) The ground-truth reconstruction using the known $\lambda=-0.2$

Figure 4. Reconstruction of a point grid on a sphere. The blue pyramids are the cameras and the black dots are the reconstructed points.

Acknowledgements The author sincerely thanks Thomas Gröninger for providing the two UAV datasets.

\section{References}

[1] S. Agarwal, N. Snavely, I. Simon, S. M. Seitz, and R. Szeliski. Building Rome in a day. In ICCV, 2009.

[2] J. H. Brito, R. Angst, K. Köser, and M. Pollefeys. Radial distortion self-calibration. In CVPR, 2013.

[3] D. Crandall, A. Owens, N. Snavely, and D. P. Huttenlocher. Discrete-continuous optimization for large-scale structure from motion. In CVPR, 2011.

[4] R. I. Hartley. Ambiguous configurations for 3-view projective reconstruction. In ECCV, pages 922-935, 2000.

[5] B. K. P. Horn. Motion fields are hardly ever ambiguous. IJCV, 1(3):259-274, 1987.

[6] F. Kahl. Critical motions and ambiguous euclidean reconstructions in auto-calibration. In ICCV, volume 1, pages 469-475, 1999.

[7] F. Kahl and R. Hartley. Critical curves and surfaces for euclidean reconstruction. In ECCV, pages 447-462, 2002.

[8] F. Kahl, R. Hartley, and K. Astrom. Critical configurations for n-view projective reconstruction. In $C V P R$, pages 158-163, 2001.

[9] F. Kahl and B. Triggs. Critical motions in euclidean structure from motion. In $C V P R$, volume 2, 1999.

[10] F. Kahl, B. Triggs, and K. Åström. Critical motions for auto-calibration when some intrinsic parameters can vary. JMIV, 13(2):131-146, 2000.
[11] H. C. Longuet-Higgins. Visual ambiguity of a moving plane. Proceedings of The Royal Society of London, Biological Sciences, pages 165-175, 1984.

[12] S. Maybank. Theory of reconstruction from image motion. 1993.

[13] S. J. Maybank. The angular velocity associated with the optical flow field due to a single moving rigid plane. In ECAI, pages 641-644, 1984.

[14] B. Mičušík and T. Pajdla. Structure from motion with wide circular field of view cameras. TPAMI, 28(7):1135-1149, 2006.

[15] N. Snavely, S. Seitz, and R. Szeliski. Photo tourism: exploring photo collections in 3D. In SIGGRAPH, pages 835-846, 2006.

[16] P. Sturm. A case against kruppa's equations for camera self-calibration. TPAMI, 22(10):1199-1204, 2000.

[17] P. F. Sturm. Critical motion sequences for monocular self-calibration and uncalibrated euclidean reconstruction. In $C V P R$, pages 1100-1105, 1997.

[18] P. F. Sturm. Critical motion sequences for the self-calibration of cameras and stereo systems with variable focal length. In BMVC, 1999.

[19] C. Wu. VisualSFM: A visual structure from motion system, 2011. http: / / ccwu.me/vsfm.

[20] C. Wu. Towards linear-time incremental structure from motion. In 3DV, 2013.

[21] C. Wu, S. Agarwal, B. Curless, and S. M. Seitz. Multicore bundle adjustment. In $C V P R, 2011$. 